\title{
Effect of Lumacaftor/lvacaftor on Pulmonary Exacerbation Rates in Members with Cystic Fibrosis in a Medicaid Population
}

\author{
Mark A. Tesell, PharmD, BCPS; Caroline J. Alper, MD; Rachel Bacon, PharmD; \\ Bonnie C. Greenwood, PharmD, BCPS; Kimberly Lenz, PharmD; Paul L. Jeffrey, PharmD; \\ and Karen Stevens, PharmD
}

\begin{abstract}
BACKGROUND: Lumacaftor/ivacaftor (LUM/IVA) is indicated for patients with cystic fibrosis (CF) homozygous for the F508del mutation in the CFTR gene. In clinical trials, LUM/IVA decreased pulmonary exacerbation rates. To our knowledge, there is no published data evaluating real-world outcomes for Medicaid patients receiving LUM/IVA.

OBJECTIVE: To compare CF pulmonary exacerbation rates before and after initiation of LUM/IVA in 1 state's Medicaid program.

METHODS: This pre-post claims analysis screened fee-for-service and managed Medicaid members who had $\geq 1$ pharmacy claim for LUM/IVA between July 2, 2015, and September 30, 2016. Members were included if they were aged $\geq 6$ years with a CF diagnosis and homozygous for the F508del mutation, consistent with the indication at study initiation. Exclusion criteria included Medicaid as a secondary payer or any break in coverage during the study. The index date was defined as the first claim for LUM/IVA. Demographics and outcomes were derived from pharmacy and medical claims. Outcomes included overall rate of pulmonary exacerbations (reported as the total events for the study population 6 months before and after the index date and average annualized rate). Pulmonary exacerbation was defined as any combination of medical claims for an emergency room (ER) visit or inpatient hospitalization with a CF pulmonary exacerbation or respiratory infection (ICD-9/10-CM codes) or pharmacy claims for an oral or intravenous antibiotic (excluding macrolides). A gap of $>7$ days was considered a new pulmonary exacerbation. Paired t-test was used to test significance.
\end{abstract}

RESULTS: 21 patients met inclusion criteria with an average age at treatment initiation of 20.1 years. Average proportion of days covered (SD) was $0.62(0.29)$. The number of pulmonary exacerbations increased from 45 to 48 during the 6 months before and after the index date, respectively, and the annualized rate increased from 4.37 to $4.66(P=0.69)$. While the number of pulmonary exacerbations associated with antibiotics alone increased (23 to 33; $P=0.08$ ), those associated with at least $1 \mathrm{ER}$ visit or inpatient hospitalization decreased (22 to $15 ; P=0.08$ ).

CONCLUSIONS: This analysis did not find a decrease in pulmonary exacerbation rate for Medicaid members receiving LUM/IVA; however, adherence was low. Further study of similar populations is needed to better understand the long-term effect of treatment.

J Manag Care Spec Pharm. 2019;25(9):1021-25

Copyright $\odot 2019$, Academy of Managed Care Pharmacy. All rights reserved.

\section{What is already known about this subject}

Lumacaftor/ivacaftor (LUM/IVA) targets the underlying cause of cystic fibrosis (CF) in patients who are homozygous for the F508del mutation.

Two randomized, placebo-controlled trials showed that treatment with LUM/IVA resulted in statistically significant individual improvements in lung function as measured by the percentage of predicted forced expiratory volume in 1 second.

Nearly $45 \%$ of patients with CF are covered by Medicaid.

\section{What this study adds}

This claims-based analysis is among the first to evaluate the real-world effect of LUM/IVA on pulmonary exacerbation rates in patients with CF in a Medicaid population.

There was no statistically significant difference observed in the rate of pulmonary exacerbation events during the 6 months before and after initiation of LUM/IVA (45-48 events, $P=0.69$ ).

After initiation of LUM/IVA, there was an increase in pulmonary exacerbations associated with antibiotic use alone (22 to 33, $P=0.08$ ) and a decrease in those associated with at least

1 emergency room visit and/or inpatient hospitalization (22 to $15 ; P=0.08)$.

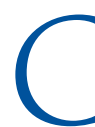
ystic fibrosis (CF) is an autosomal recessive genetic disorder caused by mutations in the CF transmembrane conductance regulator (CFTR) gene that codes for the CFTR protein. ${ }^{1}$ The CFTR protein functions as a membrane channel across cells that produce mucus, sweat, saliva, tears, and digestive enzymes. Channel transport of chloride ions into and out of cells helps control the movement of water necessary for the production of freely flowing mucus, which provides a protective coating in the airways, digestive system, reproductive system, and other organs and tissues. ${ }^{2-4}$

Although CF affects multiple organ systems, pulmonary disease is the leading cause of morbidity and mortality among patients with $\mathrm{CF} .{ }^{1}$ In addition to chest physiotherapy, medications promote airway secretion clearance. For the maintenance of lung health, recommended treatments include dornase alfa, inhaled hypertonic saline, high-dose ibuprofen, and certain antibiotics if Pseudomonas aeruginosa is persistently present. ${ }^{3}$ 
Many patients with CF experience progressive decline in lung function and episodes of acute worsening of symptoms known as pulmonary exacerbations. Pulmonary exacerbations can have a profound effect on quality of life and can cause clinical conditions such as irreversible decline of lung function and decreased survival. ${ }^{4,5}$ Mild exacerbations are often treated with oral antibiotics in the outpatient setting, while severe events result in hospitalization and intravenous antibiotic administration. ${ }^{6}$

Lumacaftor/ivacaftor (LUM/IVA) was approved by the U.S. Food and Drug Administration (FDA) on July 2, 2015, to target the underlying cause of CF in patients who are homozygous for the F508del mutation, which is the most prevalent mutation among patients in the United States. ${ }^{7}$ This agent is a combination product that contains ivacaftor, a potentiator of the CFTR protein (previously available commercially as monotherapy in a different subgroup of CF patients), as well as lumacaftor, a CFTR corrector. CFTR potentiators amplify gating function (open the chloride channel) and increase the activity of normal CFTR; CFTR correctors promote F508del protein folding (allowing movement of the protein to the cell surface). ${ }^{8}$ It is estimated that of the 30,000 U.S. individuals with CF, approximately 8,500 have 2 copies of the F508del mutation. ${ }^{9}$

LUM/IVA is indicated as an add-on therapy with other CF medications. FDA approval was primarily based on two phase 3 , randomized, double-blind, placebo-controlled trials with 1,108 patients aged 12 years or older, with predicted forced expiratory volume in 1 second $\left(\mathrm{ppFEV}_{1}\right.$ ) of 40\%-90\% normal (mean $61 \%$ ). Treatment with LUM/IVA for 24 weeks resulted in statistically significant individual improvements in percentage of ppFEV $_{1}$, ranging from 2.6 to 4.0 percentage points $(P<0.001$ for all comparisons), as well as a reduction in pulmonary exacerbations (pooled rate ratio compared with placebo $=0.61,95 \%$ confidence interval $=0.49-0.76, P<0.001$ ) Discontinuation due to adverse events was $4.2 \%$ in the treatment group versus $1.6 \%$ in the placebo group. ${ }^{10}$

Although LUM/IVA targets the underlying CF pathophysiology, it remains to be seen whether improvements demonstrated in clinical trials will be achieved in the real-world setting. Two observational studies demonstrated real-world effectiveness of LUM/IVA in improving pulmonary outcomes, measured by $\mathrm{ppFEV}_{1}$. However, rates of adverse events ranged from $39.7 \%$ to $64 \%$, and discontinuation rates ranged from $17.2 \%$ to $30 \%{ }^{11,12}$ The financial burden of managing CF for Medicaid programs, which cover nearly $45 \%$ of these patients, is significant given an approximate annual wholesale acquisition cost per patient of $\$ 260,000$, mean health care costs of $\$ 75,623$ per pulmonary exacerbation, and an average of 3.1 pulmonary exacerbations per year. ${ }^{7}$ To our knowledge, there are no published data that have evaluated real-world outcomes for Medicaid patients receiving this therapy.
The objective of this study was to compare the rate of pulmonary exacerbations before and after initiation of LUM/IVA in patients with CF in 1 state's Medicaid plan.

\section{Methods}

This observational, pre-post study used prior authorization (PA) data supplemented by pharmacy and medical claims to compare the annualized rate of pulmonary exacerbations for 6 months before and after initiation of LUM/IVA in patients with CF. Three sources were used to obtain data: an internally managed PA system, the pharmacy claims processing system, and the Medicaid management information system (medical claims). This analysis was approved by the University of Massachusetts Medical School Institutional Review Board.

The PA system contains standardized, disease-specific information collected from the PA request form, including diagnosis and mutation. The patient population included members of 1 state's fee-for-service and managed Medicaid plan with $\geq 1$ pharmacy claim for LUM/IVA (National Drug Code numbers 51167-0700-02 and 51167-0809-01) between July 2, 2015 (FDA approval date) and September 30, 2016. Patients were included if they were aged $\geq 6$ years with a diagnosis of CF and homozygous for the F508del mutation (as documented in the PA system). Exclusion criteria included having Medicaid as secondary insurer or any break in coverage during the study period.

Demographic information collected included gender, age at treatment initiation, number of $\mathrm{CF}$ medications during the 6-month pre-index period, and complications of CF. Complications, which often correlate with the burden of CF-related disease, were derived from the CF Foundation Patient Registry report and included those that could be the direct result of malfunction or deficiency of the CFTR protein or a downstream effect of the disease or its treatment. ${ }^{7}$ Diagnoses of allergic bronchopulmonary aspergillosis, arthritis, anxiety disorder, cancer, depression, diabetes, gallstones, gastroesophageal reflux disease, hearing loss, hepatic stenosis, hypertension, kidney disease, liver disease, nasal polyps, osteopenia/osteoporosis, pancreatic disease, peptic ulcer disease, renal failure requiring dialysis, and sinus disease were included following manual review of medical claims. Adherence to LUM/IVA was calculated using proportion of days covered (PDC). Members were further categorized as adherent if $\mathrm{PDC}$ was $\geq 0.8$.

Data were collected 6 months before and after the index date, which was defined as the date of the first LUM/IVA pharmacy claim. Pulmonary exacerbation was defined as any one or combination of the following events: (a) medical claim (diagnosis code in the primary or secondary position) for CF pulmonary exacerbation or respiratory infection related to an emergency room (ER) visit; (b) medical claim for CF pulmonary exacerbation or respiratory infection (International 


\begin{tabular}{|c|c|}
\hline Member Demographics & $\mathrm{N}=21$ \\
\hline Gender, female, $\mathrm{n}$ & $\mathrm{a}$ \\
\hline Age, at treatment initiation, in years, mean (range) & $20.1(12-51)$ \\
\hline Number of CF medications at baseline, mean (SD) ${ }^{\mathrm{b}}$ & $3.5(2.1)$ \\
\hline $\begin{array}{l}\text { Members receiving respiratory medications, excluding } \\
\text { respiratory antibiotics, \% }\end{array}$ & 81.8 \\
\hline Members receiving respiratory antibiotics, \% & 59.1 \\
\hline Members receiving gastrointestinal medications, $\%$ & 68.2 \\
\hline \multicolumn{2}{|l|}{ Complications of cystic fibrosis, members, \% } \\
\hline 0 & 0 \\
\hline 1 & 14.3 \\
\hline 2 & 19.0 \\
\hline$\geq 3$ & 66.7 \\
\hline PDC, mean (SD) & $0.62(0.29)$ \\
\hline $\mathrm{PDC} \geq 0.8, \mathrm{n}$ & $\mathrm{a}$ \\
\hline \multicolumn{2}{|c|}{$\begin{array}{l}\text { a Not reportable due to cell size < } 11 \text {. } \\
\text { bPharmacy claims for medications used to treat CF within } 90 \text { days of index date. } \\
\text { CF-specific medication has been defined based on the CF pulmonary guidelines as } \\
\text { dornase alfa, any inhaled antibiotic, hypertonic saline, oral macrolide antibiotics, } \\
\text { ibuprofen, leukotriene modifiers, cromolyn, inhaled beta-adrenergic receptor } \\
\text { agonists, inhaled anticholinergics, or N-acetylcysteine. } \\
C F=\text { cystic fibrosis; } P D C=\text { proportion of days covered, SD = standard deviation. }\end{array}$} \\
\hline
\end{tabular}

Classification of Diseases, Ninth Revision, Clinical Modification [ICD-9-CM] and International Classification of Diseases, Tenth Revision, Clinical Modification [ICD-10-CM] codes related to an inpatient hospitalization); or (c) pharmacy claim for an oral or intravenous antibiotic. Oral macrolides and inhaled antibiotics were excluded from this definition, since these agents are often indicated for chronic treatment. A gap of $\geq 7$ days between events was considered a new pulmonary exacerbation.

Duration of pulmonary exacerbations was defined as the number of days from the start to end date of each exacerbation. The pulmonary exacerbation start date began on the first day of a qualifying event (ER visit, inpatient admission, or antibiotic prescription fill). The end date was the inpatient discharge date, intravenous antibiotic end date, or days supply end date of the oral antibiotic supply, as long as this was followed by $\geq 7$ days in which no further claims suggesting an ongoing pulmonary exacerbation episode were observed.

The primary outcome was rate of pulmonary exacerbation per member pre- and post-index date, extrapolated to an annualized rate. Secondary outcomes included the annualized days of pulmonary exacerbations per member and a breakdown of the number and type of events that comprised each pulmonary exacerbation. Descriptive statistics were used to report demographics and outcomes. Chi-square and paired t-tests were used to test for nominal significance among categorical and continuous variables, respectively. Due to privacy concerns, cell sizes less than 11 were reported in general terms.

\section{TABLE 2 Results}

\begin{tabular}{|c|c|c|c|}
\hline $\mathrm{N}=21$ & $\begin{array}{c}\text { Before } \\
\text { LUM/IVA }\end{array}$ & $\begin{array}{c}\text { After } \\
\text { LUM/IVA }\end{array}$ & $P$ Value \\
\hline Total number of PEx & 45 & 48 & N/A \\
\hline Annualized PEx rate & 4.37 & 4.66 & $0.69^{a}$ \\
\hline Average PEx days per year & 113.31 & 125.17 & $0.55^{\mathrm{a}}$ \\
\hline \multicolumn{4}{|c|}{ Breakdown of events comprising PEx } \\
\hline Annualized ER visits & 0.87 & 0.49 & $0.21^{\mathrm{a}}$ \\
\hline $\begin{array}{l}\text { Annualized inpatient } \\
\text { hospitalizations }\end{array}$ & 2.24 & 1.55 & $0.37^{\mathrm{a}}$ \\
\hline Annualized antibiotic courses & 3.60 & 4.47 & $0.19^{a}$ \\
\hline Annualized antibiotic days & 91.64 & 108.84 & $0.42^{\mathrm{a}}$ \\
\hline $\begin{array}{l}\text { Number of PEx associated with } \\
\text { antibiotic courses only }\end{array}$ & 23 & 33 & \multirow[b]{2}{*}{$0.08^{b}$} \\
\hline $\begin{array}{l}\text { Number of PEx associated with } \\
\text { ER visit or hospitalization (with or } \\
\text { without antibiotic courses) }\end{array}$ & 22 & 15 & \\
\hline \multicolumn{4}{|c|}{$\begin{array}{l}\text { aT-test. } \\
{ }^{b} \text { Chi square. } \\
\text { ER=emergency room; LUM/IVA=lumacaftor/ivacaftor; N/A = not applicable; } \\
\text { PEx= pulmonary exacerbation. }\end{array}$} \\
\hline
\end{tabular}

\section{Results}

Twenty-one patients met criteria for study inclusion (Table 1). The majority of patients were male, and average age at treatment initiation was 20.1 years (range: 12-51). The average number of CF medications (standard deviation [SD]) per member at baseline was 3.5 (2.1). Eighty-two percent of patients received inhaled respiratory medications (excluding respiratory antibiotics), and 59\% received inhaled respiratory antibiotics such as tobramycin or aztreonam. The majority of patients (66.7\%) had $\geq 3$ complications of CF. Mean PDC was 0.62 , with a minority of patients $(<11)$ achieving a $P D C \geq 0.8$.

There was a lower, although nonsignificant, annualized pulmonary exacerbation rate before the LUM/IVA period compared with after the LUM/IVA period (4.37 vs. 4.66, respectively; $P=0.69$; Table 2 ). The average number of pulmonary exacerbation days per year increased from before the LUM/IVA period to after the LUM/IVA period (113.31 vs. 125.17 days, respectively; $P=0.55$ ).

There was a decrease from the before and after LUM/IVA periods in annualized ER visits ( 0.87 to $0.49 ; P=0.21)$ and hospitalizations (2.24 to 1.55; $P=0.37$ ); however, the number of antibiotic courses per year (3.60 to $4.47 ; P=0.19)$ and average number of antibiotic days per year increased (91.64 to 108.84; $P=0.42$ ).

When evaluated by the number and type of events that contributed to the definition of pulmonary exacerbation, the number of exacerbations associated with antibiotic courses alone increased from before the LUM/IVA period to after the LUM/IVA period (23 of 45 and 33 of 48 pulmonary exacerbations, respectively; $P=0.08$ ). However, pulmonary exacerbations associated with at least 1 ER visit and/or hospitalization 
decreased from 22 to 15 in the before and after LUM/IVA periods, respectively $(P=0.08)$.

\section{Discussion}

Although LUM/IVA has been shown in clinical trials to decrease pulmonary exacerbations, real-world data in a Medicaid population are lacking. This is especially relevant, since approximately $45 \%$ of patients with CF are Medicaid members. ${ }^{7}$ In our analysis, no significant difference was observed in the annualized rate of pulmonary exacerbations after initiation of LUM/IVA. While this is not consistent with the results of the TRAFFIC and TRANSPORT trials, it is important to note a number of differences among the studies. First, our observed rate of pulmonary exacerbations was much higher (after LUM/ IVA: 4.66 [per 365 days] vs. 0.71 in TRAFFIC and 0.67 in TRANSPORT [per 48 weeks]). ${ }^{10}$ This is likely because of a number of factors including the differences in definition of pulmonary exacerbation, as well as the potential differences in CF severity among the study populations. For inclusion, patients in the TRAFFIC and TRANSPORT studies had a low severity of illness as defined by $\mathrm{ppFEV}_{1}>40 \%$. While these data were not available for our study population, the fact that the majority of our patients had $>3$ complications of CF may be an indicator of higher disease burden. Furthermore, adherence to LUM/IVA overall was suboptimal, which may have contributed to the lack of treatment effect.

The low adherence rate in our study is consistent with other published studies that have shown a high discontinuation rate among patients receiving LUM/IVA. ${ }^{11,12}$ In 2 observational trials, $17 \%-30 \%$ of patients discontinued treatment because of adverse events. While respiratory events (e.g., cough and shortness of breath) were noted as common reasons for discontinuation, other possible causes included perceived lack of efficacy, high cost, issues with insurance coverage, or adherence difficulties. ${ }^{11,12}$ Furthermore, patients with CF are generally managed with a variety of drugs that may affect long-term respiratory function. It is possible that suboptimal engagement in CF management (e.g., adherence to other CF medications, physician visits, and pulmonary hygiene) may have influenced the change in pulmonary exacerbation.

A secondary analysis evaluated the number and type of events that corresponded to each pulmonary exacerbation. We observed an increase in the number of pulmonary exacerbations following treatment initiation that were treated solely by outpatient antibiotics. In addition, days of antibiotic treatment increased during the post-treatment period, while pulmonary exacerbations associated with at least 1 ER visit and/or hospitalization decreased. This may indicate that while LUM/IVA may not have had an effect on the incidence of pulmonary exacerbations overall, it may have contributed to a reduction in the severity of exacerbations as inferred by an increase in presumed outpatient management of the event. While a pulmonary exacerbation consists of an acute worsening of pulmonary symptoms, there can be significant heterogeneity in the degree of severity. ${ }^{13}$

Our analysis highlights the need for payers to better understand the value of novel CF disease-modifying agents based on their effects on pulmonary exacerbations for their members. A recent report from the Institute for Clinical and Economic Review evaluated CFTR therapies and found that the therapies represent a low long-term value for money, due in large part to the high cost. Specifically, the analysis recommended a discount of $71 \%-75 \%$ off the cost of LUM/IVA to align drug cost with clinical value. ${ }^{14}$ Therefore, if members receive therapy, it is important to ensure adherence and treatment benefit. This could be accomplished by medication therapy or disease state management programs to optimize medication adherence to LUM/IVA and other CF maintenance medications and by evaluating the treatment's effect on respiratory function upon PA recertification. For members who are nonadherent and/or not benefitting from CF therapies, a reevaluation of the treatment plan in collaboration with the member's prescriber could be considered.

\section{Limitations}

There are a number of limitations to address. Because patient medical charts were not available, patient specific information (e.g., disease severity) and other sources of medication (e.g., clinical trial enrollment and samples) were not evaluable. In addition, only pharmacy and medical claims were used to identify pulmonary exacerbations, an approach that has not been validated.

Despite the substantial role of pulmonary exacerbations in CF care, no consensus definition exists for these events. While our definition was consistent with other trials and incorporated a combination of ER visits, hospitalizations, and outpatient antibiotic use, applying this strategy to claims data may have resulted in a higher rate of pulmonary exacerbation. ${ }^{15-17}$ For example, because of our inability to identify the indication for an antibiotic, claims of any duration are included in our definition. This means we may have included antibiotics used for other conditions in our definition of pulmonary exacerbation.

Our annualized rate of pulmonary exacerbation was higher than that observed in clinical trials and reported by the CF Foundation Patient Registry, which may have been a result of how we defined pulmonary exacerbation. ${ }^{12}$ Furthermore, our study did not evaluate if there were changes in other components of the CF treatment regimen, besides antibiotics. For example, addition of other CF maintenance medications may have affected the rate of pulmonary exacerbations.

Another limitation of our study was duration of analysis. Cystic fibrosis is a chronic disease associated with periods of acute worsening and recovery. Therefore, it is difficult to conclusively determine the long-term effect of a treatment with just 
6 months of data. In addition, while we saw a nonsignificant difference in the opposite direction from what was expected (i.e., increase of pulmonary exacerbations after the LUM/IVA period), our study was not powered to detect the difference we observed. Because of the rarity of the condition, it is challenging to adequately power a study when drawing subjects from only 1 plan.

\section{Conclusions}

In this Medicaid program evaluation, there was no significant difference in rate of pulmonary exacerbations after initiation of LUM/IVA, although there was a low adherence rate overall. Further study in similar and larger populations is needed to better understand the long-term effect of treatment. Because of the significant cost of LUM/IVA and the cost of managing pulmonary exacerbations, payers should investigate interventions to optimize the value of this and other CF disease-modifying treatments. Frequent assessment of adherence and treatment benefit are 2 strategies that may be considered to ensure optimal use of CFTR modulator treatments and improved outcomes for patients.

\section{Authors}

MARK A. TESELL, PharmD, BCPS; CAROLINE J. ALPER, MD; RACHEL BACON, PharmD; BONNIE C. GREENWOOD, PharmD, BCPS; and KAREN STEVENS, PharmD, Clinical Pharmacy Services, UMass Medical School, Shrewsbury, Massachusetts. KIMBERLY LENZ, PharmD, and PAUL L. JEFFREY, PharmD, MassHealth, Quincy, Massachusetts.

AUTHOR CORRESPONDENCE: Mark A. Tesell, PharmD, BCPS, Consultant Pharmacist Team Lead, Clinical Pharmacy Services, UMass Medical School, 333 South St., Shrewsbury, MA 01545-7807. Tel.: 774.455.3278; E-mail: Mark.Tesell@umassmed.edu.

\section{DISCLOSURES}

No outside funding supported this study. The authors have nothing to disclose. A poster of this project was presented at the Academy of Managed Care Pharmacy Managed Care \& Specialty Pharmacy Annual Meeting 2018 in Boston, MA, on April 23-26, 2018.

\section{REFERENCES}

1. O'Sullivan BP, Freedman SD. Cystic fibrosis. Lancet. 2009;373(9678): $1891-904$
2. U.S. National Library of Medicine, Genetics Home Reference. CFTR gene. 2012. Retrieved July 6, 2018. Available at: http://ghr.nlm.nih.gov/gene/CFTR. Accessed July 12, 2019

3. Mogayzel PJ Jr, Naureckas ET, Robinson KA, et al. Cystic fibrosis pulmonary guidelines. Chronic medications for maintenance of lung health. Am J Respir Crit Care Med. 2013;187(7):680-89. Available at: https://www.atsjournals.org/doi/full/10.1164/rccm.201207-1160OE. Accessed July 12, 2019.

4. Flume PA, Mogayzel PJ, Robinson KA, et al. Cystic fibrosis pulmonary guidelines: treatment of pulmonary exacerbations. Am J Respir Crit Care Med. 2009;180(9):802-08

5. Liou TG, Adler FR, Fitzsimmons SC, Cahill BC, Hibbs JR, Marshall BC. Predictive 5-year survivorship model of cystic fibrosis. Am J Epidemiol. 2001;153(4):345-52

6. Waters V, Ratjen F. Pulmonary exacerbations in children with cystic fibrosis. Ann Am Thorac Soc. 2015;12(2):S200-06

7. Cystic Fibrosis Foundation. 2016 Patient Registry: annual data report. 2017. Available at: https://www.cff.org/Research/Researcher-Resources/ Patient-Registry/2016-Patient-Registry-Annual-Data-Report.pdf. Accessed July 12, 2019

8. Orkambi (lumacaftor/ivacaftor) tablets, for oral use. Vertex Pharmaceuticals Revised July 2019. Available at: https://pi.vrtx.com/files/uspi_lumacaftor_ ivacaftor.pdf. Accessed July 12, 2019.

9. Moskowitz SM, Chmiel JF, Sternen DL, et al. Clinical practice and genetic counseling for cystic fibrosis and CFTR-related disorders. Genet Med. 2008;10(12):851-68.

10. Wainwright CE, Elborn JS, Ramsey BW, et al. Lumacaftor-ivacaftor in patients with cystic fibrosis homozygous for Phe508del CFTR. N Eng J Med. 2015;373(3):220-31. Available at: http://www.nejm.org/doi/full/10.1056/ NEJMoal409547. Accessed July 12, 2019.

11. Jennings MT, Dezube R, Paranjape S, et al. An observational study of outcomes and tolerances in patients with cystic fibrosis initiated on lumacaftor/ivacaftor. Ann Am Thorac Soc. 2017;14(11):1662-66.

12. Hubert D, Chiron R, Camara B, et al. Real-life initiation of lumacaftor/ivacaftor combination in adults with cystic fibrosis homozygous for the Phe508del CFTR mutation and severe lung disease. J Cystic Fib. 2017;16(3):388-91.

13. Ferkol T, Rosenfeld M, Milla CE. Cystic fibrosis pulmonary exacerbations. J Pediatr. 2006;148(2):259-64.

14. Institute for Clinical Economic Review. Cystic fibrosis: final evidence report. June 7, 2018. Available at: https://icer-review.org/material/cf-finalreport/. Accessed July 12, 2019.

15. Rubin JL, Thayer S, Watkins A, Wagener JS, Hodgkins PS, Schechter MS. Frequency and costs of pulmonary exacerbations in patients with cystic fibrosis in the United States. Curr Med Res Opin. 2017;33(4):667-74.

16. Hassan M, Bonafede MM, Limone BL, Hodgkins P, Sawicki GS. The burden of cystic fibrosis in the Medicaid population. Clinicoecon Outcomes Res. 2018;10:423-31

17. VanDevanter DR, Pasta DJ, Konstan MW. Treatment and demographic factors affecting time to next pulmonary exacerbation in cystic fibrosis. J Cyst Fibros. 2015;14(6):763-69. Available at: https://www.ncbi.nlm.nih.gov/ pmc/articles/PMC4561033/. Accessed July 12, 2019. 